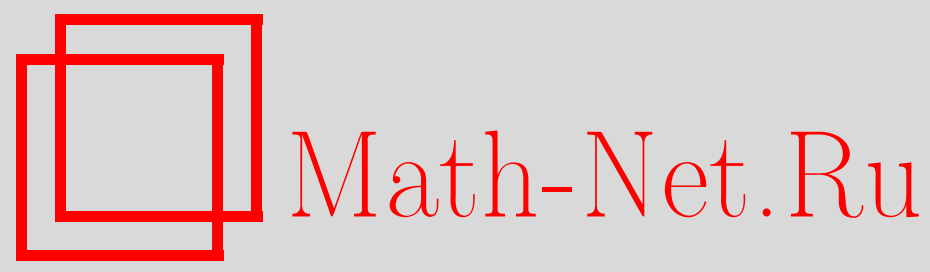

В. А. Горелов, О гипотезе Зигеля для случая линейных однородных дифференциальных уравнений второго порядка, Матем. заметки, 2004, том 75, выпуск 4, 549-565

DOI: https://doi.org/10.4213/mzm51

Использование Общероссийского математического портала Math-Net.Ru подразумевает, что вы прочитали и согласны с пользовательским соглашением http://www.mathnet.ru/rus/agreement

Параметры загрузки:

IP: 34.229 .45 .116

26 апреля 2023 г., $11: 43: 51$

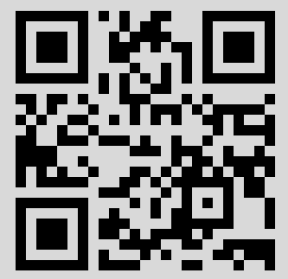




\title{
О ГИПОТЕЗЕ ЗИГЕЛЯ ДЛЯ СЛУЧАЯ ЛИНЕЙНЫХ ОДНОРОДНЫХ ДИФФЕРЕНЦИАЛЬНЫХ УРАВНЕНИЙ ВТОРОГО ПОРЯДКА
}

\section{В. А. Горелов}

\begin{abstract}
Выясняется структура множества $E$-функций, удовлетворяющих линейньм однородным дифференциальным уравнениям второго порядка с коэффициентами из $\mathbb{C}(z)$.

Библиография: 11 названий.
\end{abstract}

Введение. Метод Зигеля, опубликованньй в 1929 г. (см. [1]) и в дальнейшем получивший значительное развитие, до настояшего времени остается одним из основных методов теории трансцендентных чисел. Этот метод позволяет исследовать арифметическую природу значений некоторого класса целых аналитических функций (так называемых $E$-функций), удовлетворяющих системам линейных дифференциальных уравнений. Современное состояние метода, связанные с ним понятия и обозначения можно найти в книге [2].

В вопросе о границах применимости метода Зигеля и в вопросах его эффективности существенное значение имеет известная гипотеза Зигеля о том, что всякая $E$-функция, удовлетворяющая линейному дифференциальному уравнению с коэффициентами из $\mathbb{C}(z)$, представляется в виде многочлена с алгебраическими коэффищиентами от $z$ и конечного числа гипергеометрических $E$-функций, а также функций, получающихся из них заменой $z$ на $\alpha z$ при $\alpha \in \mathbb{A}$, где $\mathbb{A}$ - множество всех алгебраических чисел.

К гипергеометрическим $E$-функциям, в частности, относятся используемые в настоящей работе функции

$$
\varphi_{\lambda}(z)=1+\sum_{n=1}^{\infty} \frac{z^{n}}{(\lambda+1) \cdots(\lambda+n)}, \quad \lambda \neq-1,-2, \ldots,
$$

удовлетворяющие дифференциальньм уравнениям

$$
y^{\prime}=\left(1-\frac{\lambda}{z}\right) y+\frac{\lambda}{z}
$$

и функции Куммера (они же конфлюентные, или вырожденные гипергеометрические функции)

$$
A_{\mu, \nu}(z)=1+\sum_{n=1}^{\infty} \frac{\nu(\nu+1) \cdots(\nu+n-1)}{n ! \mu(\mu+1) \cdots(\mu+n-1)} z^{n}, \quad \mu \neq 0,-1, \ldots
$$


удовлетворяющие дифференциальньгм уравнениям

$$
y^{\prime \prime}+\left(-1+\frac{\mu}{z}\right) y^{\prime}-\left(\frac{\nu}{z}\right) y=0
$$

с рациональными параметрами $\lambda, \mu, \nu$.

Автору в работе [3] удалось доказать гипотезу Зигеля для случая дифференциальных уравнений первого порядка. Было установлено, что $E$-функция $f(z)$ удовлетворяет линейному дифференциальному уравнению первого порядка с коэффициентами из $\mathbb{C}(z)$ тогда и только тогда, когда она имеет вид $f(z)=P \varphi_{\lambda}(\alpha z)+P_{1}$, где $P, P_{1} \in \mathbb{A}[z], \alpha \in \mathbb{A}$, $\lambda \in \mathbb{Q}$.

Основным результатом настоящей работы является

ТЕорема 1. Е-функиия $f(z)$ удовлетворяет линейному однородному дифференииальному уравнению второго порядка с коэффиииентами из $\mathbb{C}(z)$ тогда и только тогда, когда она имеет вид

$$
f(z)=\left(P A_{\mu, \nu}(\alpha z)+P_{1} A_{\mu, \nu}^{\prime}(\alpha z)\right) e^{\alpha_{1} z},
$$

əде $P, P_{1} \in \mathbb{A}[z], \alpha, \alpha_{1} \in \mathbb{A}, \mu, \nu \in \mathbb{Q}$.

ЗАмЕчАния. 1. Если $E$-функции $f_{1}(z), f_{2}(z)$ составляют решение системы линейных однородных дифференциальных уравнений первого порядка, то каждая из них также имеет вид (2).

2. Так как $A_{\mu, \nu}^{\prime}(\alpha z)=(\nu / \mu) A_{\mu+1, \nu+1}(\alpha z)$, то для рассматриваемого в теореме случая гипотеза Зигеля справедлива.

3. Поскольку $A_{\lambda+1,1}(\alpha z)=\varphi_{\lambda}(\alpha z)$, а $\varphi_{\lambda}^{\prime}(\alpha z)=(1-\lambda /(\alpha z)) \varphi_{\lambda}(\alpha z)+\lambda /(\alpha z)$, то при $\mu=\lambda+1, \nu=1, P=P_{2}+(1-\alpha z / \lambda) P_{3}, P_{1}=(\alpha z / \lambda) P_{3}$, где $P_{2}, P_{3} \in \mathbb{A}[z]$, равенство (2) принимает вид

$$
f(z)=\left(P_{2} \varphi_{\lambda}(\alpha z)+P_{3}\right) e^{\alpha_{1} z} .
$$

В этом виде могут быть представлены функции $P(z) \in \mathbb{A}[z], \varphi_{\lambda}(z), e^{z}, \sin z=\left(e^{i z}-\right.$ $\left.e^{-i z}\right) /(2 i), \cos z, \operatorname{sh} z, \operatorname{ch} z$, всякая "неполная" гамма-функция

$$
F_{p}(z)=\int_{0}^{z} t^{p-1} e^{-t} d t=\frac{z^{p} e^{-z} \varphi_{p}(z)}{p}
$$

являющаяся целой, т.е. при $p \in \mathbb{N}$ (см. [2, с. 195, 197]). В виде (2) представляются также функции Куммера $A_{\mu, \nu}(z)$, функции Зигеля

$$
K_{\lambda}(z)=1+\sum_{n=1}^{\infty} \frac{(-1)^{n}}{n !(\lambda+1) \cdots(\lambda+n)}\left(\frac{z}{2}\right)^{2 n}, \quad \lambda \neq-1,-2, \ldots
$$

и все целые функции Бесселя $J_{p}(z)$ (при $p \in \mathbb{Z}^{+}$), так как

$$
J_{\lambda}(z)=\frac{1}{\Gamma(\lambda+1)}\left(\frac{z}{2}\right)^{\lambda} K_{\lambda}(z)=\frac{1}{\Gamma(\lambda+1)}\left(\frac{z}{2}\right)^{\lambda} e^{-i z} A_{2 \lambda+1, \lambda+1 / 2}(2 i z),
$$

где $\Gamma(z)$ - гамма-функция (см. [2, с. 212] и [4, с. 318]). Кроме того, в виде (2) могут быть представлены все целые функции Уиттекера (см. [4, с. 272]).

Следующая теорема дополняет результаты статьи [5]. 
ТЕОРема 2. При условиях теоремы 1 функиии $f(z)$ и $f^{\prime}(z)$ алгебраически зависимы (соответственно однородно алгебраически зависимы) над $\mathbb{C}(z)$ тогда и только тогда, когда $f(z)=\left(P_{\varphi_{\lambda}}(\alpha z)+P_{1}\right) e^{\sigma \alpha z}$ (соответственно $\left.f(z)=P e^{\alpha z}\right)$, где $P, P_{1} \in \mathbb{A}[z], \quad \alpha \in \mathbb{A}, \lambda, \sigma \in \mathbb{Q}$, причем если $\sigma \neq 0$, то $\lambda \in \mathbb{Z}^{+}$.

ЗАмЕчания. 1. При $\sigma \neq 0, \lambda \in \mathbb{Z}^{+}$функция $f(z)$ есть линейная комбинация показательных функций с коэффициентами из $\mathbb{A}(z)$. Например, $E$-функция $(\sin z) / z$, удовлетворяющая дифференциальному уравнению $y^{\prime \prime}+(2 / z) y^{\prime}+y=0$, представляется в виде $\left(e^{i z}-e^{-i z}\right) /(2 i z)=e^{i z} \varphi_{1}(-2 i z)=K_{1 / 2}(z)$.

2. Утверждение теоремы 2 справедливо также для алгебраически зависимых над $\mathbb{C}(z) \quad E$-функций $f_{1}(z), f_{2}(z)$, составляюших решение системы линейных однородных дифференциальных уравнений первого порядка.

1. О дифференциальных уравнениях, которым удовлетворяют $E$-функции. Пусть $\mathbb{K}$ - произвольное поле алгебраических чисел, $[\mathbb{K}: \mathbb{Q}]=h$. Поля, сопряженные с $\mathbb{K}$, будем обозначать $\mathbb{K}_{j}, j=1, \ldots, h, \mathbb{K}_{1}=\mathbb{K}$. Если $\alpha, L\left(z_{1}, \ldots, z_{m}\right)$, $P\left(z_{1}, \ldots, z_{m}\right), f(z)$ суть соответственно число из $\mathbb{K}$, линейная форма, многочлен и формальный степенной ряд с коэффициентами из $\mathbb{K}$, то через $\alpha_{[j]}, L_{[j]}\left(z_{1}, \ldots, z_{m}\right)$, $P_{[j]}\left(z_{1}, \ldots, z_{m}\right), f_{[j]}(z)$ соответственно будем обозначать число, линейную форму, многочлен и формальный степенной ряд, сопряженные с ними относительно поля $\mathbb{K}_{j}$ (см. [2, гл. $11, \S 2])$. Если $z_{m+1}=1$, то линейную форму $L\left(z_{1}, \ldots, z_{m+1}\right)$ будем назьвать линейной неоднородной формой от переменных $z_{1}, \ldots, z_{m}$.

ЛЕмма 1. Пусть совокупность формальных степенных рядов

$$
f_{k}(z)=\sum_{n=0}^{\infty} a_{k, n} z^{n}, \quad a_{k, n} \in \mathbb{K}, \quad k=1, \ldots, m, \quad m \geqslant 1,
$$

связана алгебраическим уравнением

$$
P\left(z, f_{1}(z), \ldots, f_{m}(z)\right)=0, \quad P=P\left(z, z_{1}, \ldots, z_{m}\right) \in \mathbb{C}\left[z, z_{1}, \ldots, z_{m}\right], \quad P \not \equiv 0 .
$$

Тогда

1) ряды $f_{1}(z), \ldots, f_{m}(z)$ связаны также алгебраическим уравнением

$$
\begin{gathered}
P^{*}\left(z, f_{1}(z), \ldots, f_{m}(z)\right)=0, \\
P^{*}=P^{*}\left(z, z_{1}, \ldots, z_{m}\right) \in \mathbb{Z}_{\mathbb{K}}\left[z, z_{1}, \ldots, z_{m}\right], \quad P^{*} \not \equiv 0,
\end{gathered}
$$

причем если некоторый числовой коэффициент в многочлене $P^{*}$ отличен от нуля, то соответствующий коэффициент в $P$ также отличен от ну$\Omega я ;$

2) для любого такого многочлена $P^{*}$ и для любого $j, 1 \leqslant j \leqslant h$,

$$
P_{[j]}^{*}\left(z,\left(f_{1}\right)_{[j]}(z), \ldots,\left(f_{m}\right)_{[j]}(z)\right)=0 .
$$

ДокАЗАТЕльство. Будем рассматривать все ненулевые числовые коэффициенты многочлена $P$ как неопределенные. Рассуждая аналогично доказательству леммы $2 \oint 2$ гл. 3 книги [2], получим, что эти неопределенные коэффициенты удовлетворяют системе из конечного числа линейных однородных уравнений с коэффициентами из $\mathbb{K}$. Поэтому искомые коэффициенты могут быть выбраны из $\mathbb{Z}_{\mathbb{K}}$, и первое утверждение леммы доказано. Для доказательства второго утверждения леммы заметим, что при повторении прежних рассуждений для сопряженных рядов $\left(f_{1}\right)_{[j]}(z), \ldots,\left(f_{m}\right)_{[j]}(z)$ мы получим систему линейных уравнений, сопряженных с прежними. Следовательно, каждое ее решение также будет сопряженным с прежним. Лемма доказана. 
ЛЕмма 2. Пусть КЕ-функиия (см. [2]) $f(z)$ является решением линейного дифференииального уравнения

$$
Q_{m} y^{(m)}+Q_{m-1} y^{(m-1)}+\cdots+Q_{0} y=Q, \quad m \geqslant 1,
$$

с коэффициентами из $\mathbb{C}[z]$, взаимно простыми в совокупности, и не удовлетворяет никакому линейному дифференииальному уравнению меньшего порядка с коәффициентами из $\mathbb{C}[z]$ (или, при $Q \equiv 0,-$ никакому линейному однородному дифференциальному уравнению меньшего порядка с коэффициентами из $\mathbb{C}[z])$. Тогда

1) коэффициенты уравнения (4) определены однозначно с точностью до множителя из $\mathbb{C}$ и могут быть выбраны из $\mathbb{K}[z] ;$

2) при выборе коэффициентов уравнения (4) из $\mathbb{K}[z]$ всякая сопряэсенная функиия $f_{[j]}(z), 1 \leqslant j \leqslant h$, удовлетворяет дифференииальному уравнению

$$
\left(Q_{m}\right)_{[j]} y^{(m)}+\left(Q_{m-1}\right)_{[j]} y^{(m-1)}+\cdots+\left(Q_{0}\right)_{[j]} y=(Q)_{[j]} .
$$

ДОКАЗАТЕЛЬСТВо. При условиях леммы функция $f(z)$, очевидно, линейно независима над $\mathbb{C}[z]$ с $f^{\prime}(z), \ldots, f^{(m-1)}(z)$ и 1 , а в однородном случае - c $f^{\prime}(z), \ldots, f^{(m-1)}(z)$. Поэтому рациональные функции $Q_{k} / Q_{m}$ определяются однозначно, а многочлены $Q$, $Q_{0}, \ldots, Q_{m}-$ с точностью до множителя из $\mathbb{C}$. Отсюда, применяя лемму 1, получаем утверждение леммы 2.

Лемма 3. Пусть выполнены условия леммы 2, а $\xi \in \mathbb{A} \backslash\{0\}$. Тогда существуют числа $r_{1}, \ldots, r_{m} \in \mathbb{Z}^{+}$такие, что для произвольной линейной неоднородной (в однородном случае - линейной) формы $L\left(z_{1}, \ldots, z_{m}\right) \not \equiv 0$ с коэффициентами из $\mathbb{Z}_{\mathbb{K}}$ размера $H$ и произвольного $\varepsilon>0$ выполняется неравенство

$$
\max _{1 \leqslant j \leqslant h}\left|L_{[j]}\left(f_{[j]}^{\left(r_{1}\right)}\left(\xi_{[j]}\right), \ldots, f_{[j]}^{\left(r_{m}\right)}\left(\xi_{[j]}\right)\right)\right|>b H^{\delta},
$$

где $\delta=-m-\varepsilon($ в однородном случае $\delta=1-m-\varepsilon), \quad b>0-$ постоянная, не зависящая от $H$.

ДокАЗАтЕльство. Разложим функцию $f(z)$, а также многочлены $Q_{k}$ в степенные ряды по степеням $\zeta=z-\xi$ :

$$
\begin{gathered}
f(z)=\sum_{\nu=0}^{\infty} a_{\nu} \zeta^{\nu}, \quad Q_{k}=q_{k, 0}+q_{k, 1} \zeta+\cdots+q_{k, \mu} \zeta^{\mu} \\
Q=q_{0}+q_{1} \zeta+\cdots+q_{\omega} \zeta^{\omega}, \quad q_{j}, q_{k, l} \in \mathbb{A}, \quad \mu=\max \operatorname{deg} Q_{k} .
\end{gathered}
$$

При подстановке этих разложений в уравнение (4) и приравнивании в нем коэффициентов при степенях $\zeta^{\nu-m}$ получается линейное рекуррентное соотношение для тейлоровских коэффициентов $a_{\nu}$ функции $f(z)$. Чтобы найти явньй вид этого соотношения, заметим, что применение дифференциального оператора $q_{k, l} \zeta^{l}(d / d \zeta)^{k}, 0 \leqslant k \leqslant m$, $0 \leqslant l \leqslant \mu, \mathrm{k}$ члену ряда $a_{\nu-s} \zeta^{\nu-s}, s \in \mathbb{Z}$, дает одночлен степени $\nu-s-k+l$. Поскольку $\nu-s-m \leqslant \nu-s-k+l \leqslant \nu-s+\mu$, то для получения одночлена, содержащего $\zeta^{\nu-m}$, необходимо условие $0 \leqslant s \leqslant m+\mu$. При вьполнении этого условия одночлен степени $\nu-m$ можно получить из члена ряда $a_{\nu-s} \zeta^{\nu-s}$, применяя любой дифференциальньй оператор $q_{m-j, s-j} \zeta^{s-j}(d / d \zeta)^{m-j}$, где $0 \leqslant j \leqslant \min (s, m)$. Коэффициент у полученного одночлена будет равен $a_{\nu-s} q_{m-j, s-j}(\nu-s) \cdots(\nu-s-m+j+1)$, если $j \leqslant m-1$, и 
$a_{\nu-s} q_{0, s-m}$, если $j=m$ (в этом случае $s \geqslant m$ ). Складьвая эти коэффициенты, находим искомое рекуррентное соотношение:

$$
\begin{aligned}
\sum_{s=0}^{m-1} a_{\nu-s} & \sum_{j=0}^{s} q_{m-j, s-j} \prod_{i=0}^{m-1-j}(\nu-s-i) \\
& +\sum_{s=m}^{m+\mu} a_{\nu-s}\left(\sum_{j=0}^{m-1} q_{m-j, s-j} \prod_{i=0}^{m-1-j}(\nu-s-i)+q_{0, s-m}\right)=q_{\nu-m} .
\end{aligned}
$$

Если положить $a_{\nu}=0$ при $\nu<0, q_{k, l}=0$ при $l<0$ и $l>\mu, q_{\nu}=0$ при $\nu<0$ и $\nu>\omega$, то равенство (7) справедливо для всех $\nu \in \mathbb{Z}$. Запишем равенство (7) в виде

$$
p_{s_{0}}\left(\nu-s_{0}\right) a_{\nu-s_{0}}+p_{s_{0}+1}\left(\nu-s_{0}\right) a_{\nu-s_{0}-1}+\cdots+p_{m+\mu}\left(\nu-s_{0}\right) a_{\nu-m-\mu}=q_{\nu-m},
$$

что равносильно

$$
p_{s_{0}}(r) a_{r}+p_{s_{0}+1}(r) a_{r-1}+\cdots+p_{m+\mu}(r) a_{r-m-\mu+s_{0}}=q_{r-m+s_{0}},
$$

где $p_{s}(x) \in \mathbb{A}[x], \operatorname{deg} p_{s}(x) \leqslant m$, а $s_{0}$ - наименьшее из $s$ таких, что $\sum_{j=0}^{s}\left|q_{m-j, s-j}\right| \neq 0$. Поскольку $Q_{m} \not \equiv 0$, то $s_{0} \leqslant \min (m, \mu)$. Как следует из равенства $(7)$,

$$
p_{s_{0}}(x)=\sum_{j=0}^{\min \left(s_{0}, m-1\right)} q_{m-j, s_{0}-j} \prod_{i=0}^{m-1-j}(x-i)+q_{0, s_{0}-m} .
$$

При условиях леммы $E$-функция $f(z)$ не является многочленом (в однородном случае $f(z) \not \equiv 0)$. Поэтому степень трансцендентности (в однородном случае однородной трансцендентности) множества функций $f(z), f^{\prime}(z), \ldots, f^{(m-1)}(z)$ не меньше 1 . Для любого $r \in \mathbb{Z}^{+}$обозначим $\mathbf{L}_{r}$ линейное пространство над $\mathbb{A}$, порожденное числами $a_{0}, a_{1}, \ldots, a_{r}$ и 1 (в однородном случае $\left.-a_{0}, a_{1}, \ldots, a_{r}\right)$, и пусть $\mathbf{L}_{-1}=\mathbb{A}$ (в однородном случае $\left.\mathbf{L}_{-1}=\{0\}\right)$. Если $m_{1}=\max \operatorname{dim} \mathbf{L}_{r}$, то, как следует из теоремы 1 работы [3] и соотношения (8), $1 \leqslant m_{1}<\infty$. Обозначим через $r_{1,1}$ наименьший из индексов $r \in \mathbb{Z}^{+}$со свойством $a_{r} \notin \mathbf{L}_{r-1}, r_{1,2}-$ второй по величине индекс с таким свойством и так далее до $r_{1, m_{1}}$. Подставляя в равенство (8) любое $r=r_{1, t}, 1 \leqslant t \leqslant m_{1}$, получим, что $p_{s_{0}}\left(r_{1, t}\right)=0,-$ иначе число $a_{r_{1, t}}$ линейно зависело бы от чисел $a_{r} \mathrm{c} r<r_{1, t}$ и, следовательно, принадлежало бы $\mathbf{L}_{r_{1, t}-1}$. Вообще, поскольку всякая функция $f_{[j]}(z)$, $1 \leqslant j \leqslant h$, является $E$-функцией, удовлетворяющей уравнению $(5)$, то коэффициенты $a_{j, r}$ ее степенного разложения по степеням $\left(z-\xi_{[j]}\right)$ удовлетворяют рекуррентному соотношению

$$
\left(p_{s_{0}}\right)_{[j]}(r) a_{j, r}+\left(p_{s_{0}+1}\right)_{[j]}(r) a_{j, r-1}+\cdots+\left(p_{m+\mu}\right)_{[j]}(r) a_{j, r-m-\mu+s_{0}}=\left(q_{r-m+s_{0}}\right)_{[j]},
$$

а многочлен $\left(p_{s_{0}}\right)_{[j]}(r)$ имеет не менее $m_{j} \geqslant 1$ попарно различных цельх неотрицательных корней $r_{j, 1}, \ldots, r_{j, m_{j}}$. Положим

$$
\left\{x_{1}, \ldots, x_{m_{0}}\right\}=\bigcup_{j=1}^{h}\left\{r_{j, 1}, \ldots, r_{j, m_{j}}\right\}
$$

Поскольку рациональные корни многочлена не меняются при алгебраическом сопряжении и $\operatorname{deg} p_{s_{0}}(x) \leqslant m$, то все числа $x_{1}<x_{2}<\cdots<x_{m_{0}}$ являются корнями многочлена $p_{s_{0}}(r)$, а $1 \leqslant m_{0} \leqslant m$. При этом не исключается, что многочлен $p_{s_{0}}(r)$ может 
иметь и другие корни из $\mathbb{Z}^{+}$. Очевидно, что для любого $j, 1 \leqslant j \leqslant h$, среди чисел $f_{[j]}^{\left(x_{1}\right)}\left(\xi_{[j]}\right), \ldots, f_{[j]}^{\left(x_{m_{0}}\right)}\left(\xi_{[j]}\right)$ имеются линейно независимые и потому отличные от нуля.

Если функции

$$
f^{\left(x_{1}\right)}(z), \quad \ldots, \quad f^{\left(x_{m_{0}}\right)}(z)
$$

линейно зависимы с 1 (в однородном случае - линейно зависимы) над $\mathbb{C}(z)$, то согласно лемме 1 найдутся многочлены $A_{1}(z), \ldots, A_{m_{0}}(z), A(z) \in \mathbb{Z}_{\mathbb{K}}[z]$, взаимно простые в совокупности и не все тождественно равные нулю (в однородном случае $A(z) \equiv 0)$, такие, что

$$
\left(A_{m_{0}}\right)_{[j]}(z) f_{[j]}^{\left(x_{m_{0}}\right)}(z)+\cdots+\left(A_{1}\right)_{[j]}(z) f_{[j]}^{\left(x_{1}\right)}(z)+A_{[j]}(z) \equiv 0, \quad j=1, \ldots, h .
$$

Пусть $l$ - наибольший из индексов, для которых $A_{l}(\xi) \neq 0$. Так как $\left(A_{l}\right)_{[j]}\left(\xi_{[j]}\right) \neq 0$ для любого $j, 1 \leqslant j \leqslant h$, то отсюда следует, что число $a_{j, r_{l}}$ линейно зависимо с $a_{j, r_{l}-1}, \ldots, a_{j, 0}$ и 1 (в однородном случае - с $a_{j, r_{l}-1}, \ldots, a_{j, 0}$ ). Но при некотором $j$ это противоречит выбору $r_{j, 1}, \ldots, r_{j, m_{j}}$. Следовательно, функции (9) линейно независимы с 1 (в однородном случае - линейно независимы) над $\mathbb{C}(z)$. В однородном случае дополним множество этих функций до базиса в линейном пространстве над $\mathbb{C}(z)$, порожденном функциями

$$
f(z), \quad f^{\prime}(z), \ldots, \quad f^{(k)}(z), \ldots
$$

Элементы этого базиса также выберем из (10) и обозначим $g_{1}(z), \ldots, g_{m}(z)$. Общий случай сводится к однородному введением функции $g_{0}(z) \equiv 1$. Как и при доказательстве леммы 8 работы [3], для любого достаточно большого $n \in \mathbb{N}$ и $\varepsilon>0$ построим $m$ линейно независимых линейных форм

$$
L_{k}=b_{k, 1} g_{1}(\xi)+\cdots+b_{k, m} g_{m}(\xi), \quad b_{k, \lambda} \in \mathbb{Z}_{\mathbb{K}}, \quad k, \lambda=1, \ldots, m
$$

с условием

$$
\begin{gathered}
\max _{1 \leqslant j \leqslant h}\left|\left(b_{k, 1}\right)_{[j]}\left(g_{1}\right)_{[j]}\left(\xi_{[j]}\right)+\cdots+\left(b_{k, m}\right)_{[j]}\left(g_{m}\right)_{[j]}\left(\xi_{[j]}\right)\right|=O\left(n^{-(m-1-\varepsilon) n}\right), \\
\overline{\left|b_{k, \lambda}\right|}=O\left(n^{(1+\varepsilon) n}\right), \quad k, \lambda=1, \ldots, m .
\end{gathered}
$$

Среди этих линейных форм можно выбрать $m-1$ форм, линейно независимых с формой $L\left(g_{1}(\xi), \ldots, g_{m}(\xi)\right)$. Кроме того, согласно доказанному выше для любого $j, 1 \leqslant j \leqslant h$, среди чисел $\left(g_{1}\right)_{[j]}\left(\xi_{[j]}\right), \ldots,\left(g_{m}\right)_{[j]}\left(\xi_{[j]}\right)$ найдутся отличные от нуля. Далее дословно повторяем рассуждения доказательства теоремы 1 гл. 11 книги [2].

ЛЕмма 4. При условиях леммы 2 всякая особая точка $\xi \neq 0$ дифференииального уравнения (4) есть регулярная особая точка соответствующего однородного уравнения.

ДокАЗАТЕЛЬСТВО. Пусть кроме корней $x_{1}, \ldots, x_{m_{0}}$, существование которых установлено при доказательстве леммы 3 , многочлен $p_{s_{0}}(r)$ имеет и другие целые неотрицательные корни. Обозначим их $d_{1}, \ldots, d_{n}$. Тогда $m_{0}+n \leqslant \operatorname{deg} p_{s_{0}}(r) \leqslant m$. Предположим, что функции

$$
f^{\left(d_{1}\right)}(z), \quad \ldots, \quad f^{\left(d_{n}\right)}(z), \quad f^{\left(x_{m_{0}}\right)}(z), \quad \ldots, \quad f^{\left(x_{1}\right)}(z)
$$


линейно зависимы с 1 (в однородном случае - линейно зависимы) над $\mathbb{C}(z)$. Рассмотрим все подмножества совокупности функций (11), содержашие множество (9) и связанные только одним линейным уравнением вида

$$
\begin{aligned}
& B_{1}(z) f^{\left(d_{i_{1}}\right)}(z)+\cdots+B_{k}(z) f^{\left(d_{i_{k}}\right)}(z) \\
& \quad+B_{k+1}(z) f^{\left(x_{m_{0}}\right)}(z)+\cdots+B_{k+m_{0}}(z) f^{\left(x_{1}\right)}(z)+B(z) \equiv 0
\end{aligned}
$$

где $1 \leqslant k \leqslant n, d_{i_{1}}>d_{i_{2}}>\cdots>d_{i_{k}}$, а $B_{1}(z), \ldots, B_{k+m_{0}}(z), B(z)$ - взаимно простые в совокупности многочлены из $\mathbb{Z}_{\mathbb{K}}[z]$, не все тождественно равные нулю (в однородном случае $B(z) \equiv 0)$. Пусть $l$ - наименьший из индексов, для которых $B_{l}(\xi) \neq 0$. Тогда $\left(B_{l}\right)_{[j]}\left(\xi_{[j]}\right) \neq 0$ для любого $j, 1 \leqslant j \leqslant h$. Следовательно, $l \leqslant k, \mathrm{a}$

$$
f_{[j]}^{\left(d_{i_{l}}\right)}\left(\xi_{[j]}\right)=L_{[j]}\left(f_{[j]}^{\left(d_{i_{l+1}}\right)}\left(\xi_{[j]}\right), \ldots, f_{[j]}^{\left(d_{i_{k}}\right)}\left(\xi_{[j]}\right), f_{[j]}^{\left(x_{m_{0}}\right)}\left(\xi_{[j]}\right), \ldots, f_{[j]}^{\left(x_{1}\right)}\left(\xi_{[j]}\right)\right),
$$

где $L\left(z_{1}, \ldots, z_{m_{0}+k-l}\right)$ - некоторая линейная неоднородная (в однородном случае - линейная) форма с коэффициентами из $\mathbb{K}$. Если $d$-максимум величин $d_{i_{l}}$, стоящих в левых частях уравнений (12), то исключим функцию $f^{(d)}(z)$ из множества $(11)$. Допустим, что после этого в нем останутся только линейно независимые с 1 (в однородном случае - линейно независимые) функции

$$
f^{\left(d_{k_{1}}\right)}(z), \ldots, f^{\left(d_{k_{n-1}}\right)}(z), f^{\left(x_{m_{0}}\right)}(z), \ldots, f^{\left(x_{1}\right)}(z) .
$$

Тогда, повторяя рассуждения леммы 3 , достроим множество (13) до базиса $g_{1}(z), \ldots$, $g_{m}(z)$. Одна из этих функций, положим для определенности $g_{m}(z)=f^{(M)}(z)$, не принадлежит множеству (11). Равенство (8) при $r=M$ имеет вид $L^{*}=L^{*}\left(g_{m}(\xi)\right.$, $\left.f^{(M-1)}(\xi), \ldots, f(\xi)\right)=0$, где $L^{*}\left(z_{1}, \ldots, z_{M+1}\right)$ - линейная неоднородная (в однородном случае - линейная) форма с коэффициентами из $\mathbb{K}$, причем ее коэффициент при $g_{m}(\xi)$ есть $p_{s_{0}}(M) \neq 0$, так как $M \notin\left\{x_{1}, \ldots, x_{m_{0}}, d_{1}, \ldots, d_{n}\right\}$. Поскольку те из чисел $f^{(M-1)}(\xi), \ldots, f(\xi)$, которые не являются значениями функций (13), можно представить как линейные комбинации значений функций (13), и эти представления сохраняются при алгебраическом сопряжении, то

$$
L^{*}=L^{* *}\left(f^{(M)}(\xi), f^{\left(d_{k_{1}}\right)}(\xi), \ldots, f^{\left(d_{k_{n-1}}\right)}(\xi), f^{\left(x_{m_{0}}\right)}(\xi), \ldots, f^{\left(x_{1}\right)}(\xi)\right)=0
$$

где $L^{* *}\left(z_{1}, \ldots, z_{m_{0}+n}\right) \not \equiv 0$ - линейная неоднородная (в однородном случае - линейная) форма с коэффициентами из $\mathbb{K}$, причем

$$
L_{[j]}^{* *}\left(f_{[j]}^{(M)}\left(\xi_{[j]}\right), f_{[j]}^{\left(d_{k_{1}}\right)}\left(\xi_{[j]}\right), \ldots, f_{[j]}^{\left(d_{k_{n-1}}\right)}\left(\xi_{[j]}\right), f_{[j]}^{\left(x_{m_{0}}\right)}\left(\xi_{[j]}\right), \ldots, f_{[j]}^{\left(x_{1}\right)}\left(\xi_{[j]}\right)\right)=0,
$$

где $j=1, \ldots, h$. Полученное противоречие с неравенством (6) показывает, что функции (13) линейно зависимы с 1 (в однородном случае - линейно зависимы). Но тогда, повторяя предыдущие рассуждения с функциями (13) вместо (11), получим множество из $m_{0}+n-2$ функций. Вновь повторяя, при необходимости, эти рассуждения, неизбежно придем к множеству линейно независимых функций и противоречию с неравенством (6). Противоречия можно избежать лишь при условии, что функции (11) линейно независимы с 1 (в однородном случае - линейно независимы), $m_{0}+n=m=\operatorname{deg} p_{s_{0}}(r)$. Поэтому $q_{m, s_{0}} \neq 0, \operatorname{ord}_{z=\xi} Q_{m}=s_{0}$, а многочлен $p_{s_{0}}(r)$ имеет $m$ попарно различных целых неотрицательных корней. Из определения $s_{0}$ получаем, что $q_{k, l}=0$ при $l<k-m+s_{0}$ 
и, следовательно, $\operatorname{ord}_{z=\xi} Q_{k} \geqslant k-m+s_{0}$. Тогда для любого $k, 0 \leqslant k<m$, порядок полюса функции $Q_{k} / Q_{m}$ в точке $\xi$ равен

$$
\operatorname{ord}_{z=\xi} Q_{m}-\operatorname{ord}_{z=\xi} Q_{k} \leqslant s_{0}-\left(k-m+s_{0}\right)=m-k,
$$

что и доказьвает лемму.

Показателями дифференциального уравнения (4) (или, если $Q \not \equiv 0$, соответствующего однородного дифференциального уравнения) в точке $z=\xi$ назьваются корни так назьваемого определяющего уравнения $F(r)=0$. В случае регулярной особой точки, умножая дифференциальное уравнение (4) на нужную степень $(z-\xi)$, можно представить его коэффициенты в виде

$$
Q_{k}^{*}=(z-\xi)^{k} h_{k}, \quad h_{k} \in \mathbb{A}[z-\xi], \quad k=0,1, \ldots, m, \quad h_{m}(\xi) \neq 0 .
$$

Тогда определяющее уравнение имеет вид

$$
F(r)=r(r-1) \cdots(r-m+1) h_{m}(\xi)+\cdots+r h_{1}(\xi)+h_{0}(\xi)=0
$$

(см., например, [6, п. 18.1]). Если функция

$$
y=(z-\xi)^{r} \sum_{\nu=0}^{\infty} a_{\nu}(z-\xi)^{\nu}, \quad a_{0} \neq 0, \quad r \in \mathbb{C}
$$

есть решение линейного однородного дифференциального уравнения, то $r$ - корень его определяющего уравнения в точке $\xi$ (см. там же).

Легко видеть, что при условиях леммы $3 h_{m}(\xi)=q_{m, s_{0}}, h_{m-1}(\xi)=q_{m-1, s_{0}-1}, \ldots$, a $F(r) \equiv p_{s_{0}}(r)$. В конце доказательства леммы 4 установлено, что многочлен $p_{s_{0}}(r)$ имеет ровно $m$ попарно различных корней из $\mathbb{Z}^{+}$. Тем самым доказана

ЛЕмма 5. При условиях леммы 3 показатели однородного дифференииального уравнения, соответствующего уравнению (4), в точке $z=\xi$ суть $m$ попарно различных целых неотрицательных чисел.

Если точка $z=\xi$ является регулярной особой точкой дифференциального уравнения, то отсюда, вообще говоря, не следует, что его решения в этой точке также имеют особенности. Докажем, что в нашем случае реализуется именно эта возможность, т.е. что всякая особая точка $\xi \neq 0$ является так назьваемой кажущейся особенностью.

Лемма 6. При условиях леммы 3 всякое решение уравнения (4), а также соответствующего ему однородного дифференииального уравнения, голоморфно в точке $z=\xi$.

ДокАЗАТЕЛЬСТво. Пусть $r_{1}<r_{2}<\cdots<r_{m}$ - показатели однородного дифференциального уравнения, соответствующего (4), в точке $\xi$. Докажем, что оно имеет фундаментальную систему решений, состоящую из функций вида

$$
y=\sum_{\nu=r}^{\infty} a_{\nu}(z-\xi)^{\nu}, \quad a_{r} \neq 0
$$

где $r$ пробегает множество $\left\{r_{1}, \ldots, r_{m}\right\}$. Коэффициенты $a_{\nu}$ для $i$-го ряда, $1 \leqslant i \leqslant m$, выберем следующим образом. В качестве $a_{r_{i}}, \ldots, a_{r_{m}}$ возьмем произвольные величины, $\mathrm{a}$ 
для $\nu \notin\left\{r_{i}, \ldots, r_{m}\right\}$, поскольку $p_{s_{0}}(\nu) \neq 0$, величину $a_{\nu}$ найдем из рекуррентного соотношения (8), положив в нем $q_{j}=0$. Докажем, что при условиях леммы равенство (8), где считаем $q_{j}=0$, справедливо и для $r=r_{k}, i \leqslant k \leqslant m$. Действительно, равенству (8) удовлетворяют тейлоровские коэффициенты функции $f(z)$. При $r=r_{k}$ оно будет иметь вид $L=L\left(f^{\left(r_{k}-1\right)}(\xi) /\left(r_{k}-1\right) !, \ldots, f(\xi)\right)=0$, где $L\left(z_{1}, \ldots, z_{r_{k}}\right)$ - линейная неоднородная (в однородном случае - линейная) форма с коэффициентами из $\mathbb{K}$. Те числа $f^{(r)}(\xi) / r$ !, у которых $r \notin\left\{r_{k-1}, \ldots, r_{1}\right\}$, с помощью равенства (8) линейно выражаются через $f^{\left(r_{k-1}\right)}(\xi) /\left(r_{k-1}\right) !, \ldots, f^{\left(r_{1}\right)}(\xi) /\left(r_{1}\right)$ !. Таким образом, $L=L^{*}\left(f^{\left(r_{k-1}\right)}(\xi) /\left(r_{k-1}\right) !\right.$, $\left.\ldots, f^{\left(r_{1}\right)}(\xi) /\left(r_{1}\right) !\right)$, где $L^{*}\left(z_{1}, \ldots, z_{k-1}\right)$ - линейная неоднородная (в однородном случае - линейная) форма с коэффициентами из $\mathbb{K}$, причем

$$
L_{[j]}^{*}\left(\frac{f_{[j]}^{\left(r_{k-1}\right)}\left(\xi_{[j]}\right)}{\left(r_{k-1}\right) !}, \ldots, \frac{f_{[j]}^{\left(r_{1}\right)}\left(\xi_{[j]}\right)}{\left(r_{1}\right) !}\right)=0, \quad j=1, \ldots, h .
$$

Согласно лемме $3 L^{*}\left(z_{1}, \ldots, z_{k-1}\right) \equiv 0$. Для однородного дифференциального уравнения, соответствующего (4), построенная линейная форма будет совпадать с однородной частью линейной формы $L^{*}\left(z_{1}, \ldots, z_{k-1}\right)$ и также будет тождественно равна нулю. Но тогда, очевидно, при замене чисел $f^{\left(r_{1}\right)}(\xi) /\left(r_{1}\right) !, \ldots, f^{\left(r_{m}\right)}(\xi) /\left(r_{m}\right) !$ на произвольные величины $a_{r_{1}}, \ldots, a_{r_{m}}$ равенства $L=0$ и (8) останутся справедливыми. Следовательно, ряды (15) формально удовлетворяют однородному дифференциальному уравнению, соответствующему (4) и в силу сходимости таких рядов в окрестности регулярной особой точки (см. $[7$, п. 16.2]) и их линейной независимости мы получаем искомую фундаментальную систему решений. А так как общее решение уравнения (4) есть сумма $E$-функции $f(z)$ и общего решения соответствуюшего однородного уравнения, то оно также голоморфно в точке $\xi$. Лемма доказана.

ЛЕмма 7. Если при условиях леммы 3 выполнено равенство $Q_{m}(\xi)=0$, то

$$
\frac{Q_{k}}{Q_{m}}=\frac{c_{k}}{(z-\xi)^{m-k}}+\frac{\psi_{k}(z)}{(z-\xi)^{m-k-1}}, \quad k=0,1, \ldots, m-1,
$$

где $c_{k} \in \mathbb{Z},-c_{m-1} \in \mathbb{N},(-1)^{m} c_{0} \in \mathbb{Z}^{+}, \psi_{k}(z) \in \mathbb{A}(z), \psi_{k}(\xi) \neq \infty$.

ДокАЗАтЕльство. Так как $Q_{k} / Q_{m}=Q_{k}^{*} / Q_{m}^{*}=(z-\xi)^{-(m-k)} h_{k} / h_{m}$, то $c_{k}=$ $h_{k}(\xi) / h_{m}(\xi)$, и уравнение (14) можно переписать в виде

$$
\frac{F(r)}{h_{m}(\xi)}=r(r-1) \cdots(r-m+1)+r(r-1) \cdots(r-m+2) c_{m-1}+\cdots+r c_{1}+c_{0}=0 .
$$

Последовательно рассматривая в этом уравнении одночлены, содержашие $r^{k}, k=m-1$, $m-2, \ldots, 0$, убеждаемся, что каждое число $c_{k}$ выражается в виде линейной комбинации коэффициентов многочлена $F(r) / h_{m}(\xi)$ и чисел $c_{k+1}, \ldots, c_{m-1}$ с целыми коэффициентами. Так как многочлен $F(r) / h_{m}(\xi)$ имеет целые неотрицательные корни $r_{1}, \ldots, r_{m}$, то все его коэффициенты и, следовательно, $c_{0}, \ldots, c_{m-1}$ являются целыми числами. Кроме того, $(-1)^{m} c_{0}=r_{1} \cdots r_{m} \geqslant 0$, а $c_{m-1}=1+2+\cdots+(m-1)-\left(r_{1}+\cdots+r_{m}\right) \leqslant 0$. Предположим, что $c_{m-1}=0$. Тогда $r_{j}=j-1, j=1, \ldots, m$. Рассмотрим однородное уравнение, соответствующее уравнению (4). Согласно доказательству леммы 6 фундаментальную систему решений этого уравнения можно составить из функций $y_{j}=(z-\xi)^{j-1} \omega_{j}, j=1, \ldots, m$, где $\omega_{j}-$ функции, голоморфные и не равныенулю в точке $z=\xi$. Пусть наибольший порядок полюсов функций $Q_{m-1} / Q_{m}, \ldots, Q_{0} / Q_{m}$ в точке $z=\xi$ равен $p, 0 \leqslant p \leqslant m$, a $l$ - наибольший из индексов таких, что функция $Q_{l} / Q_{m}$ 
имеет в точке $z=\xi$ полюс порядка $p$. Тогда, разделив однородное уравнение, соответствующее (4), на $Q_{m}$ и подставив в него функцию $y_{l+1}$, получим, что порядок полюса $\left(Q_{l} / Q_{m}\right) y_{l+1}^{(l)}$ равен $p$, а порядок полюсов предыдущих и последующих слагаемых меньше $p$. Полученное противоречие показывает, что точка $z=\xi$ не является особой точкой однородного уравнения, соответствующего (4), и, следовательно, самого уравнения (4). Лемма доказана.

ЛЕмма 8. Пусть при условиях леммы 2 функции $f(z), f^{\prime}(z), \ldots, f^{(m-1)}(z)$ алгебраически (в однородном случае - однородно алгебраически) независимы над $\mathbb{C}(z)$. Тогда в однородном случае наименьиий показатель дифференииального уравнения (4) в точке $z=\xi \in \mathbb{A} \backslash\{0\}$ совпадает $c \operatorname{ord}_{z=\xi} f(z)$, а в общем случае наименьиий показатель однородного дифференциального уравнения, соответствуюшего (4), равен $\max _{z=\xi}(f(z)-P(z))$, где максимум берется по всем многочленам $P(z) \in \mathbb{A}[z]$.

ДокАЗАТЕЛЬСТВо. Пусть $r_{1}<r_{2}<\cdots<r_{m}$ - показатели однородного дифференциального уравнения, соответствуюшего (4), в точке $\xi$. В случае $Q \equiv 0$ порядок нуля функции $f(z)$ в точке $\xi$ равен одному из $r_{k}$. Но поскольку производные функции $f(z)$ порядков $r_{1}, \ldots, r_{m}$ в точке $\xi$ однородно алгебраически независимы, то $f^{\left(r_{1}\right)}(\xi) \neq 0$ и $\operatorname{ord}_{z=\xi} f(z)=r_{1}$. В случае $Q \not \equiv 0$ из рекуррентного соотношения (8) получаем, что значения в точке $\xi$ производных функции $f(z)$ до порядка $r_{1}-1$ включительно суть алгебраические числа, а значение производной порядка $r_{1}$ трансцендентно. Следовательно, разность функции $f(z)$ и произвольного многочлена с алгебраическими коэффициентами может иметь порядок нуля, не превосходящий $r_{1}$. Лемма доказана.

Лемма 9. Пусть выполнены условия леммы 8, а $P(z)$ - многочлен с алгебраическими коэффициентами, все корни которого являются нулями функиии $f(z)$. Тогда $g(z)=f(z) / P(z)$ есть Е-функиия.

ДокАЗАТЕЛЬСтво. Очевидно, что функция $g(z)$ целая, а лемму достаточно доказать для случая $P(z)=z-\xi, \xi \in \mathbb{A} \backslash\{0\}, Q_{m}(\xi)=0$. Пусть разложения функций $f(z)$ и $g(z)$ в ряд Тейлора имеют вид

$$
f(z)=a_{0}+a_{1} z+\cdots+\frac{a_{n}}{n !} z^{n}+\cdots, \quad g(z)=b_{0}+b_{1} z+\cdots+\frac{b_{n}}{n !} z^{n}+\cdots .
$$

Тогда из равенства

$$
f(z)=(z-\xi) g(z)
$$

следует, что для любого $n \in \mathbb{N}$

$$
a_{0}=-\xi b_{0}, \quad a_{1}=b_{0}-\xi b_{1}, \quad \ldots, \quad \frac{a_{n}}{n !}=\frac{b_{n-1}}{(n-1) !}-\frac{\xi b_{n}}{n !} .
$$

Умножая эти равенства соответственно на $1, \xi, \ldots, \xi^{n}$ и складывая, получим

$$
\frac{b_{n}}{n !} \xi^{n+1}=-\left(a_{0}+a_{1} \xi+\cdots+\frac{a_{n}}{n !} \xi^{n}\right), \quad n=0,1, \ldots
$$

Пусть $\left\{d_{n}\right\}$ - последовательность натуральных чисел такая, что $d_{n} a_{k} \in \mathbb{Z}_{A}, k=0$, $1, \ldots, n, n=1,2, \ldots$, и $d_{n}=O\left(n^{\varepsilon n}\right)$ при $n \rightarrow \infty$. Тогда, умножая равенства (17) на $n !$, получим, что существует последовательность $\kappa_{n}=d_{n}(\operatorname{den}(1 / \xi))^{n+1}$ (здесь $\operatorname{den} \alpha-$ знаменатель алгебраического числа $\alpha$ ) со свойствами $\kappa_{n} \in \mathbb{N}, \kappa_{n} b_{k} \in \mathbb{Z}_{\mathbb{A}}, k=0,1, \ldots, n$, $\kappa_{n}=O\left(n^{\varepsilon n}\right), n=1,2, \ldots$ 
Докажем теперь, что

$$
\max _{1 \leqslant j \leqslant h}\left|\left(b_{n}\right)_{[j]}\right|=O\left(n^{\varepsilon n}\right),
$$

где $h=[\mathbb{K}: \mathbb{Q}], \mathbb{K}$ - наименьшее поле алгебраических чисел, содержащее $\xi$ и коэффициенты $a_{n} / n$ ! разложения функции $f(z)$. Согласно второму утверждению леммы 1 из равенства (16) получаем

$$
f_{[j]}(z)=\left(z-\xi_{[j]}\right) g_{[j]}(z), \quad j=1, \ldots, h .
$$

Заметим, что $f_{[j]}(z)$ является $E$-функцией. Согласно лемме 2 она удовлетворяет уравнению (5). Так как $f(\xi)=0$, то ввиду леммы 8 все показатели однородного уравнения, соответствующего (4), в точке $z=\xi$ больше нуля. Показатели однородного уравнения, соответствующего (5), в точке $z=\xi_{[j]}$ являются корнями определяющего уравнения, сопряженного с (14). Поэтому, будучи цельми числами, они не меняются при алгебраическом сопряжении и также больше нуля. Отсюда в однородном случае согласно лемме 8 получаем $f_{[j]}\left(\xi_{[j]}\right)=0$. В общем случае из равенства $(8)$ имеем $p_{s_{0}}(0) f(\xi)=q_{s_{0}-m}=0$, откуда также получаем

$$
f_{[j]}\left(\xi_{[j]}\right)=\left(\frac{q_{s_{0}-m}}{p_{s_{0}}(0)}\right)_{[j]}=0 .
$$

Из определения $E$-функций и условий доказьваемой леммы следует, что $f_{[j]}(z)$, как и $f(z)$, является целой функцией первого порядка. Тогда ввиду равенства $(18) g_{[j]}(z)$ также является целой функцией первого порядка. Отсюда следуют оценки $\left|\left(b_{n}\right)_{[j]}\right|=$ $O\left(n^{\varepsilon n}\right), j=1, \ldots, h$. Лемма доказана.

2. Доказательство теоремы 2. Всякая $E$-функция $f(z)$ является либо целой трансцендентной функцией первого порядка, либо многочленом. Если $f(z) \in \mathbb{A}[z]$, то утверждение теоремы справедливо. Поэтому всюду в дальнейшем будем считать, что $f(z)$ - трансцендентная функция, удовлетворяющая уравнению

$$
y^{\prime \prime}+Q_{1} y^{\prime}+Q_{0} y=0, \quad Q_{1}, Q_{0} \in \mathbb{A}(z) .
$$

Наличие среди решений уравнения (19) целой функции первого порядка накладывает ограничения на рост коэффициентов $Q_{1}$ и $Q_{0}$. Они могут быть установлены с помощью следующего утверждения, доказанного в более сильном варианте Стрелицом [8] (см. также [9, гл. 5, п. 2]).

Лемма 10. Пусть $g(z)$ - функиия, имеющая однозначный модуль в некоторой окрестности бесконечности, удовлетворяет уравнению (19), а ее порядок, определяемый так же, как и порядок иелой функиии, равен $\rho$. Пусть лорановскими разложениями коэффициентов уравнения (19) являются

$$
Q_{1}=z^{q_{1}} \sum_{\nu=0}^{\infty} \frac{a_{\nu}}{z^{\nu}}, \quad Q_{0}=z^{q_{0}} \sum_{\nu=0}^{\infty} \frac{b_{\nu}}{z^{\nu}}, \quad q_{0}, q_{1} \in \mathbb{Z}, \quad a_{0} b_{0} \neq 0,
$$

причем не имеет место случай $q_{1} \leqslant-1, q_{0} \leqslant-2$ (т.е. случай регулярной особой точки в бесконечности, когда, очевидно, всякое челое решение есть многочлен). Тогда

1) если $q_{0} \geqslant 2 q_{1}$, mo $\rho=1+q_{0} / 2$;

2) если $q_{0}<q_{1}$, то 
а) возможно решение $g(z)=z^{\mu} \psi(z)$, где $\mu \in \mathbb{C}, \quad \psi(z)$ голоморфна в бесконечности;

б) для всех остальных решений $\rho=1+q_{1}$;

3) если $q_{1}-1<q_{0}<2 q_{1}$, то

а) возмохно решение $g(z)=z^{\mu} \exp \left(-\lambda_{0} z^{q_{0}-q_{1}+1}+\cdots+\lambda\right) \psi(z)$, где $\mu \in \mathbb{C}$, $\psi(z)$ голоморфна в бесконечности;

б) для всех остальных решений $\rho=1+q_{1}$.

Поскольку $\rho=1$, то случай 3 ) возможен лишь при $q_{0}=q_{1}$. Тогда $f(z)=P(z) e^{\alpha z}$, где $P(z) \in \mathbb{A}(z), \alpha \in \mathbb{A}$, и утверждение теоремы выполняется. То, что случай 3 ) действительно может иметь место, показьвает пример уравнения $y^{\prime \prime}+z y^{\prime}+(z-2+2 / z) y=0$, которому удовлетворяет $E$-функция $z e^{-z}$. Случаи 1$)$ и 2$)$ возможны лишш при $q_{0} q_{1}=0$, $q_{0}, q_{1} \in \mathbb{Z}^{-}$.

Следовательно, при доказательстве теоремы можно ограничиться рассмотрением дифференциального уравнения (19) вида

$$
y^{\prime \prime}+\left(a+\frac{Q_{1}}{Q}\right) y^{\prime}+\left(b+\frac{Q_{0}}{Q}\right) y=0, \quad(a, b) \neq(0,0),
$$

где $a, b \in \mathbb{A}, Q_{0}, Q_{1}, Q \in \mathbb{A}[z], \max \left(\operatorname{deg} Q_{1}, \operatorname{deg} Q_{0}\right)<\operatorname{deg} Q$.

Следуюшие две леммы доказьваются непосредственным вычислением.

Лемма 11. Пусть $f, Q_{0}, Q_{1}, P$ - произвольные аналитические функиии, причем функиия $f$ удовлетворяет дифференииальному уравнению

$$
y^{\prime \prime}+Q_{1} y^{\prime}+Q_{0} y=0 .
$$

Тогда функиия $g=P f$ удовлетворяет дифференииальному уравнению

$$
y^{\prime \prime}+\left(Q_{1}-\frac{2 P^{\prime}}{P}\right) y^{\prime}+\left(Q_{0}-\frac{P^{\prime \prime}}{P}-\left(Q_{1}-\frac{2 P^{\prime}}{P}\right) \frac{P^{\prime}}{P}\right) y=0 .
$$

СлЕДСТвИЕ. При условиях леммы 11 функиия $g=e^{\alpha z} f$, где $\alpha \in \mathbb{C}$, удовлетворяет дифференциальному уравнению

$$
y^{\prime \prime}+\left(Q_{1}-2 \alpha\right) y^{\prime}+\left(Q_{0}-\alpha Q_{1}+\alpha^{2}\right) y=0 .
$$

ЛЕмма 12. Пусть $f, Q_{0}, Q_{1}, Q$ - произвольные аналитические функции, причем функция $f$ удовлетворяет дифференциальному уравнению

$$
y^{\prime \prime}+\frac{Q_{1}}{Q} y^{\prime}+\frac{Q_{0}}{Q} y=0 .
$$

Тогда функиия $g=f^{\prime}$ удовлетворяет дифференциальному уравнению

$$
y^{\prime \prime}+\left(\frac{Q_{1}}{Q}+\frac{Q^{\prime}}{Q}-\frac{Q_{0}^{\prime}}{Q_{0}}\right) y^{\prime}+\left(\frac{Q_{0}}{Q}+\frac{Q_{1}^{\prime}}{Q}-\frac{Q_{0}^{\prime}}{Q_{0}} \frac{Q_{1}}{Q}\right) y=0 .
$$

Лемма 13 (см. [2, гл. 6, лемма 2]). Пусть функиия $y_{0} \not \equiv 0$ удовлетворяет уравнению (20) и алгебраччески зависима с $y_{0}^{\prime}$ над $\mathbb{C}(z)$. Тогда существует решение $y_{*}$ уравнения (20) такое, что его логарифмическая производная $y_{*}^{\prime} / y_{*}$ есть алгебраическая функиия над $\mathbb{C}(z)$. 
ЛЕмма 14. При условиях теоремы 2 существует решение $v_{*}$ уравнения (19) mакое, что $v_{*}^{\prime} / v_{*}=P / S, P, S \in \mathbb{C}[z]$.

ДокАЗАТЕЛЬСТво. Если функции $f=f(z)$ и $f^{\prime}$ однородно алгебраически зависимы над $\mathbb{C}(z)$, то $f^{\prime} / f \in \mathbb{C}(z)$ и утверждение леммы вьполняется. Если $f$ и $f^{\prime}$ однородно алгебраически независимы над $\mathbb{C}(z)$, то рассмотрим функцию $g=\exp (a z / 2) y_{*}$, где $y_{*}-$ peшение уравнения (20), о котором говорится в лемме 13 . Функции $g$ и $\exp (a z / 2) f$ согласно следствию из леммы 11 удовлетворяют уравнению $y^{\prime \prime}+\left(Q_{1} / Q\right) y^{\prime}+\left(b^{*}+Q_{2} / Q\right) y=0$, где $b^{*} \in \mathbb{A}, Q_{1}, Q_{2}, Q \in \mathbb{A}[z], \max \left(\operatorname{deg} Q_{1}, \operatorname{deg} Q_{2}\right)<\operatorname{deg} Q$. Если $b^{*}=0$, то, как следует из леммы $10, \exp (a z / 2) f \in \mathbb{A}[z]$ и все доказано. Пусть $b^{*} \neq 0$. Рассмотрим функцию $u=g^{\prime} / g=y_{*}^{\prime} / y_{*}+a / 2$. Она является алгебраической и удовлетворяет уравнению Риккати

$$
u^{\prime}+u^{2}+\frac{Q_{1}}{Q} u+b^{*}+\frac{Q_{2}}{Q}=0, \quad Q, Q_{1}, Q_{2} \in \mathbb{A}[z]
$$

где $b^{*} \in \mathbb{A} \backslash\{0\}, \max \left(\operatorname{deg} Q_{1}, \operatorname{deg} Q_{2}\right)<\operatorname{deg} Q$. Докажем, что $z=\infty$ не является точкой ветвления функции $u$. Отсюда и из леммы 6 будет следовать, что эта функция не имеет точек ветвления вообще.

Разложим функцию $u$ в окрестности точки $z=\infty$ в степенной ряд с рациональньми показателями:

$$
u=\sum_{k=0}^{\infty} c_{k} z^{r_{k}}, \quad c_{0} \neq 0, \quad r_{0}>r_{1}>r_{2}>\cdots
$$

Подставив разложение $(22)$ в уравнение $(21)$, убеждаемся, что $r_{0}=0, c_{0}^{2}=-b^{*}$. Если не все показатели $r_{k}$ в ряду (22) целые, то пусть $r_{k_{1}}$ - наибольший дробньй показатель такой, что $c_{k_{1}} \neq 0$. Тогда, подставляя разложение $(22)$ в уравнение $(21)$ и рассматривая коэффищиент при наибольшей дробной степени $z$, получаем $c_{0} c_{k_{1}}=0$, что противоречит нашим допушениям. Следовательно, $u \in \mathbb{C}(z)$ и лемма доказана.

Представим функцию $f$ в виде

$$
f=v_{*} \int w d z
$$

Тогда функция $w$ должна удовлетворять дифференциальному уравнению $w^{\prime}+(a+$ $\left.Q_{1} / Q+2 P / S\right) w=0$ (см., например, [7, с. 164]). Дифференцируя равенство (23), получаем

$$
f^{\prime}=\left(\frac{P}{S}\right) f+v_{*} w,
$$

откуда следует, что $v_{*} w S$ есть $E$-функция. Она удовлетворяет однородному дифференциальному уравнению первого порядка

$$
y^{\prime}=\left(\frac{S^{\prime}}{S}-a-\frac{Q_{1}}{Q}-\frac{P}{S}\right) y
$$

и, следовательно, $v_{*} w S=R e^{\alpha z}$, где $R \in \mathbb{A}[z], \alpha \in \mathbb{A}$ (см. [2, с. 189]). Тогда $v_{*} w=$ $e^{\alpha z} R / S$ и, подставляя эту функцию в уравнение $(24)$, находим, что $E$-функция $e^{-\alpha z} f$ удовлетворяет уравнению

$$
y^{\prime}=\left(\frac{P}{S}-\alpha\right) y+\frac{R}{S} .
$$


Но тогда согласно теореме 2 работы [3] $e^{-\alpha z} f=P_{1} \varphi_{\lambda}\left(\alpha_{1} z\right)+P_{2}$, откуда

$$
f=\left(P_{1} \varphi_{\lambda}\left(\alpha_{1} z\right)+P_{2}\right) e^{\alpha z}, \quad f^{\prime}=\frac{\left(P_{3} \varphi_{\lambda}\left(\alpha_{1} z\right)+P_{4}\right) e^{\alpha z}}{z}
$$

где $P_{1}, \ldots, P_{4} \in \mathbb{A}[z], \alpha_{1} \in \mathbb{A}$. Рассмотрим функцию

$$
F=P_{1} f^{\prime}-P_{3} \frac{f}{z}=\frac{e^{\alpha z}\left(P_{1} P_{4}-P_{2} P_{3}\right)}{z}
$$

В случае $F \equiv 0$ или $P_{1} \equiv 0$ имеем $f^{\prime} / f \in \mathbb{C}(z)$ и теорема справедлива. Если же $F \not \equiv 0$, $P_{1} \not \equiv 0$, то из условий теоремы 2 получаем, что функции $f$ и $F$ алгебраически зависимы над $\mathbb{C}(z)$, а отсюда следует алгебраическая зависимость функций $e^{\alpha z}$ и $\varphi_{\lambda}\left(\alpha_{1} z\right)$. Согласно теореме 2 гл. 5 книги [2] это возможно лишш в случаях $\alpha \alpha_{1}=0$ и $\lambda \in \mathbb{Z}^{+}$. Но если $\lambda=k \in \mathbb{Z}^{+}$, то $\varphi_{\lambda}(z)=k !\left(e^{z}-\sum_{n=0}^{k-1} z^{n} / n !\right) / z^{k}$ (см. [2, гл. $\left.\left.5, \S 2\right]\right)$, откуда

$$
f=R_{1} e^{\alpha_{1} z}+R_{2} e^{\alpha_{2} z}, \quad f^{\prime}=R_{3} e^{\alpha_{1} z}+R_{4} e^{\alpha_{2} z}
$$

где $R_{1}, \ldots, R_{4} \in \mathbb{C}(z), \alpha_{1}, \alpha_{2} \in \mathbb{A}$. Повторяя предыдущие рассуждения, получим, что функции $e^{\alpha_{1} z}$ и $e^{\alpha_{2} z}$ алгебраически зависимы над $\mathbb{C}(z)$ и, следовательно, числа $\alpha_{1}, \alpha_{2}$ линейно зависимы. Теорема 2 доказана.

\section{3. Доказательство теоремы 1.}

ЛЕмма 15 (см., например, [7, с. 526]). Пусть в дифференииальном уравнении (19)

$$
Q_{1}=\frac{c_{1}}{z-\xi}+\psi_{1}(z), \quad Q_{0}=\frac{c_{0}}{(z-\xi)^{2}}+\frac{\psi_{0}(z)}{z-\xi}
$$

где $\xi \in \mathbb{C}, \psi_{1}(z), \psi_{0}(z)-$ функиии, голоморфные в точке $z=\xi$. Тогда сумма и произведение показателей дифференциального уравнения (19) в точке $\xi$ равны соответственно $1-c_{1} и c_{0}$.

ЛЕмма 16. Пусть $f$ есть Е-функиия, $a \theta \in \mathbb{A}[z, d / d z]-$ дифференциальный оператор минимального порядка, аннулируюший $f$. Тогда если $z=0$ - особая точка дифференциального уравнения $\theta f=0$, то она является регулярной особой точкой.

Лемма 16 следует из более сильных утверждений, полученных Ивом Андрэ (см. [10, c. 724 и 746]). Отметим, что этим же путем можно получить аналоги лемм 4 и 6 для однородного случая.

ЛЕмма 17. Пусть Е-функиия $f(z)$ удовлетворяет дифференциальному уравнению

$$
y^{\prime \prime}+\left(a+\frac{a_{1}}{z}\right) y^{\prime}+\left(b+\frac{b_{1}}{z}\right) y=0, \quad a, a_{1}, b, b_{1} \in \mathbb{A} .
$$

Тогда $f(z)$ либо имеет вид (3), либо $f(z)=c z^{l} e^{-\alpha z} A_{\beta, \gamma}((2 \alpha-a) z), a_{1}, \beta, \gamma \in \mathbb{Q}$, $c \in \mathbb{A}, \quad l \in \mathbb{Z}^{+}, \alpha^{2}-a \alpha+b=0$, причем $l=0$ при $a_{1} \notin \mathbb{Z}^{-}$. 
ДокАЗАТЕЛЬСТво. Если $b \neq 0$, рассмотрим функцию $g(z)=e^{\alpha z} f(z)$. Согласно следствию из леммы 11 эта функция удовлетворяет уравнению

$$
y^{\prime \prime}+\left(a-2 \alpha+\frac{a_{1}}{z}\right) y^{\prime}+\frac{b_{1}-\alpha a_{1}}{z} y=0,
$$

аналогичному исходному, но уже с $b=0$. Если $a=2 \alpha$, то, как следует из леммы 10 , $g(z)$ есть многочлен и все доказано. Если $a \neq 2 \alpha$, то рассмотрим функцию $g_{1}(z)=$ $g(z /(2 \alpha-a))$. Она удовлетворяет уравнению

$$
y^{\prime \prime}+\left(-1+\frac{a_{1}}{z}\right) y^{\prime}-\left(\frac{\gamma}{z}\right) y=0, \quad \gamma=\frac{\alpha a_{1}-b_{1}}{2 \alpha-a} .
$$

Предположим, что уравнение (26) имеет два целых решения, линейно независимых над $\mathbb{C}$. Тогда общее решение уравнения (26) является однозначньп и $g_{1}(z)$ представляется в виде

$$
R_{1} e^{\alpha_{1} z}+R_{2} e^{\alpha_{2} z}=\left(R_{1} e^{\left(\alpha_{1}-\alpha_{2}\right) z}+R_{2}\right) e^{\alpha_{2} z},
$$

где $R_{1}, R_{2} \in \mathbb{C}(z)$ (см. [6, п. 18.6], или $\left[7\right.$, п. 15.5]). Но тогда $E$-функция $g_{1}(z) e^{-\alpha_{2} z}=$ $R_{1} e^{\left(\alpha_{1}-\alpha_{2}\right) z}+R_{2}$ удовлетворяет линейному дифференциальному уравнению первого порядка с коэффищиентами из $\mathbb{C}(z)$. Согласно теореме 2 работы [3] имеет место разложение $g_{1}(z) e^{-\alpha_{2} z}=P \varphi_{\lambda}(\alpha z)+P_{1}$, где $P, P_{1} \in \mathbb{A}[z]$, и лемма справедлива. Предположим теперь, что уравнение (26) имеет, с точностью до множителя из $\mathbb{C}$, только одно целое решение. Тогда при $a_{1} \notin \mathbb{Z}^{-}$это решение есть функция Куммера и, следовательно, $g(z /(2 \alpha-a))=c A_{a_{1}, \gamma}(z), c \in \mathbb{C}$. Отсюда получаем, что $f(z)=c e^{-\alpha z} A_{a_{1}, \gamma}((2 \alpha-a) z)$, $c \in \mathbb{A}$ и $A_{a_{1}, \gamma}((2 \alpha-a) z)$ есть $E$-функция. Но тогда $a_{1}, \gamma \in \mathbb{Q}$ или выполняется равенство (3) (см. [11] и [2, с. 228]). Если же $a_{1} \in \mathbb{Z}^{-}$, то согласно лемме $11 g(z /(2 \alpha-a))=$ $c z^{1-a_{1}} A_{2-a_{1}, \gamma-a_{1}+1}(z)$ и лемма также справедлива.

Перейдем теперь непосредственно к доказательству теоремы 1. Достаточность ее условий следует из того, что функция $f(z)$, заданная равенством $(2)$, линейно зависима с $f^{\prime}(z)$ и $f^{\prime \prime}(z)$ над $\mathbb{C}(z)$. Для доказательства необходимости, как уже было установлено, можно ограничиться рассмотрением случая, когда функция $f(z)$ удовлетворяет дифференциальному уравнению (20) и однородно алгебраически независима с $f^{\prime}(z)$ над $\mathbb{C}(z)$. Обозначим $\xi_{1}, \ldots, \xi_{m}, \xi_{0}=0$ все конечные особые точки уравнения $(20)$. Пусть функция $f(z)$ имеет в точке $\xi_{j}, 0 \leqslant j \leqslant m$, нуль кратности $n_{j} \geqslant 0$. Рассмотрим многочлен $P=z^{n_{0}}\left(z-\xi_{1}\right)^{n_{1}} \cdots\left(z-\xi_{m}\right)^{n_{m}}$ и функцию $g(z)=f(z) / P$. Согласно леммам 9 , $11,4,16 g(z)$ является $E$-функцией и удовлетворяет дифференциальному уравнению вида (20), не имеющему особых точек, отличных от $\xi_{0}, \xi_{1}, \ldots, \xi_{m}$, причем всякая особая точка является регулярной. Поскольку $g\left(\xi_{j}\right) \neq 0, j=0,1, \ldots, m$, то один из показателей дифференциального уравнения в каждой точке $\xi_{j}$ равен нулю. Отсюда ввиду леммы 15 получаем, что функция $Q_{0} / Q$ может иметь полюсы не более чем первого порядка. Тогда из леммы 7 следует, что функция $g(z)$ удовлетворяет либо дифференциальному уравнению (25), либо дифференциальному уравнению

$$
y^{\prime \prime}+\left(a-\frac{a_{0}}{z}-\frac{k_{1}}{z-\xi_{1}}-\cdots-\frac{k_{m}}{z-\xi_{m}}\right) y^{\prime}+\left(b+\frac{b_{0}}{z}+\frac{b_{1}}{z-\xi_{1}}+\cdots+\frac{b_{m}}{z-\xi_{m}}\right) y=0
$$

где $a, a_{0}, b, b_{0}, b_{1}, \ldots, b_{m} \in \mathbb{A}, k_{j} \in \mathbb{N}, \xi_{j} \in \mathbb{A}, j=1, \ldots, m$.

Пусть $k=k_{1}+\cdots+k_{m}$. Индукцией по $k$ докажем, что все $E$-функции, удовлетворяюшие уравнениям вида $(27)$ и, следовательно, $g(z)$ представляются в виде

$$
\left(R A_{\mu, \nu}(\alpha z)+R_{1} A_{\mu, \nu}^{\prime}(\alpha z)\right) e^{\alpha_{1} z},
$$


где $R, R_{1} \in \mathbb{A}(z), \alpha, \alpha_{1} \in \mathbb{A}, \mu, \nu \in \mathbb{Q}$. Если $k=0$, то данное утверждение вьполняется согласно лемме 17. Предположим, что утверждение справедливо для всех $E$-функций, удовлетворяющих произвольным дифференциальным уравнениям вида (27) при любых $m \in \mathbb{Z}^{+}, k \leqslant n-1, n \geqslant 1$, и докажем его для $k=n$. Если $b_{m} \neq 0$, рассмотрим функцию $g_{1}(z)=\exp \left(-b_{m} z / k_{m}\right) g(z)$. Согласно следствию из леммы 11 функция $g_{1}(z)$ удовлетворяет дифференциальному уравнению

$$
\begin{aligned}
y^{\prime \prime}+ & \left(a^{*}-\frac{a_{0}}{z}-\frac{k_{1}}{z-\xi_{1}}-\cdots-\frac{k_{m}}{z-\xi_{m}}\right) y^{\prime} \\
& +\left(b^{*}+\frac{b_{0}^{*}}{z}+\frac{b_{1}^{*}}{z-\xi_{1}}+\cdots+\frac{b_{m-1}^{*}}{z-\xi_{m-1}}\right) y=0
\end{aligned}
$$

где $a^{*}, b^{*}, b_{0}^{*}, \ldots, b_{m-1}^{*} \in \mathbb{A}$, которое можно записать в виде

$$
y^{\prime \prime}+\frac{Q_{2}}{z\left(z-\xi_{1}\right) \cdots\left(z-\xi_{m}\right)} y^{\prime}+\frac{Q_{3}}{z\left(z-\xi_{1}\right) \cdots\left(z-\xi_{m}\right)} y=0,
$$

где $Q_{2}, Q_{3} \in \mathbb{A}[z], \operatorname{deg} Q_{2} \leqslant m+1$. Если $Q_{3} \equiv 0$, то $g_{1}(z)=R e^{\alpha z}, R \in \mathbb{A}[z], \alpha \in \mathbb{A}$, и доказьваемое утверждение справедливо. Если $Q_{3} \not \equiv 0$, то $Q_{3}=z^{l_{0}}\left(z-\xi_{1}\right)^{l_{1}} \cdots \times$ $\left(z-\xi_{m}\right)^{l_{m}} Q_{4}, Q_{4} \in \mathbb{A}[z], l_{j} \geqslant 0, l_{m} \geqslant 1, \operatorname{deg} Q_{4}=t \leqslant m+1-\left(l_{0}+l_{1}+\cdots+l_{m}\right)$, $Q_{4}=c\left(z-\beta_{1}\right)^{t_{1}} \cdots\left(z-\beta_{s}\right)^{t_{s}}, t_{i} \in \mathbb{N}, \beta_{i} \neq \beta_{j}, \beta_{i} \neq \xi_{j}, c \in \mathbb{A} \backslash\{0\}$. Согласно лемме 12 функция $g_{1}^{\prime}(z)$ удовлетворяет уравнению

$$
\begin{aligned}
y^{\prime \prime}+ & \left(a^{*}+\frac{1-a_{0}-l_{0}}{z}-\sum_{j=1}^{m} \frac{k_{j}-1+l_{j}}{z-\xi_{j}}-\sum_{i=1}^{s} \frac{t_{i}}{z-\beta_{i}}\right) y^{\prime} \\
& +\left(\frac{a_{0} l_{0}}{z^{2}}+\sum_{j=1}^{m} \frac{k_{j} l_{j}}{\left(z-\xi_{j}\right)^{2}}+\frac{Q_{5}}{z\left(z-\xi_{1}\right) \cdots\left(z-\xi_{m}\right)\left(z-\beta_{1}\right) \cdots\left(z-\beta_{s}\right)}\right) y=0
\end{aligned}
$$

где $Q_{5} \in \mathbb{A}[z]$. Показатели дифференциального уравнения $(30)$ в точке $\xi_{j} \neq 0$ согласно лемме 15 равны $l_{j}$ и $k_{j}$ (в точке $\xi_{0}$ - соответственно $l_{0}$ и $a_{0}$ ). Ввиду леммы 8 порядок нуля функции $g_{1}^{\prime}(z)$ в точке $\xi_{j} \neq 0$ равен $l_{j}^{*}=\min \left(l_{j}, k_{j}\right), l_{m}^{*} \geqslant 1$. Рассмотрим функцию $g_{2}(z)=g_{1}^{\prime}(z) / P_{1}(z)$, где $P_{1}(z)=z^{l_{0}^{*}}\left(z-\xi_{1}\right)_{1}^{l_{1}^{*}} \cdots\left(z-\xi_{m}\right)^{l_{m}^{*}}, l_{0}^{*}=\operatorname{ord}_{z=0} g_{1}^{\prime}(z)$. Согласно леммам 9, 11 и $15 g_{2}(z)$ является $E$-функцией, удовлетворяющей дифференциальному уравнению, коэффициент которого при $y$ имеет полюсы не более чем первого порядка, а коэффициент при $y^{\prime}$ равен

$$
a^{*}+\frac{1-a_{0}-l_{0}+2 l_{0}^{*}}{z}-\sum_{j=1}^{m} \frac{k_{j}-1+l_{j}-2 l_{j}^{*}}{z-\xi_{j}}-\sum_{i=1}^{s} \frac{t_{i}}{z-\beta_{i}} .
$$

Величина $k$ у полученного дифференциального уравнения не превосходит $n+t-m+$ $\left(l_{1}+\cdots+l_{m}\right)-2\left(l_{1}^{*}+\cdots+l_{m}^{*}\right) \leqslant n+1-2\left(l_{1}^{*}+\cdots+l_{m}^{*}\right) \leqslant n-1$. Согласно индуктивному предположению функция $g_{2}(z)$ и, следовательно, $g_{1}^{\prime}(z)$, имеет вид $(28)$. Но тогда из уравнения (29), которому удовлетворяет функция $g_{1}(z)$, получаем, что $g_{1}(z)$ и $g(z)$ также имеют вид (28). Поскольку $g(z)=f(z) / P(z)$, то тем самым доказана представимость функции $f(z)$ в виде $(28)$ или, что равносильно, в виде

$$
f(z)=\left(\frac{P_{2} A_{\mu, \nu}(\alpha z)+P_{3} A_{\mu, \nu}^{\prime}(\alpha z)}{P_{4}}\right) e^{\alpha_{1} z},
$$


где $P_{2}, P_{3}, P_{4}$ - многочлены с алгебраическими коэффициентами, взаимно простые в совокупности. Предположим, что многочлен $P_{4}$ имеет корень $\xi \neq 0$. Тогда из равенства (31) следует однородная алгебраическая зависимость чисел $A_{\mu, \nu}(\alpha \xi), A_{\mu, \nu}^{\prime}(\alpha \xi)$. Согласно первой основной теореме Шидловского (см. [2, гл. 3]) отсюда получаем, что функции $A_{\mu, \nu}(z), A_{\mu, \nu}^{\prime}(z)$ должны быть однородно алгебраически зависимыми над $\mathbb{C}(z)$. Но тогда функция $A_{\mu, \nu}(z)$ удовлетворяет линейному однородному дифференциальному уравнению первого порядка и поэтому $A_{\mu, \nu}(z)=P_{5} e^{\alpha_{2} z}, P_{5} \in \mathbb{A}[z]$. Из равенства (31) $f(z)=R_{2} e^{\alpha_{3} z}$, где $R_{2} \in \mathbb{A}(z)$. Но поскольку $f(z)$ есть $E$-функция, то $R_{2} \in \mathbb{A}[z]$ и утверждение теоремы вьполняется. Осталось разобрать случай, когда $P_{4}=z^{n}, n \in \mathbb{N}$. Тогда равенство (31) имеет вид

$$
\begin{aligned}
f(z)= & \left(\left(\frac{p_{-n}}{z^{n}}+\cdots+p_{0}+\cdots+p_{t} z^{t}\right) A_{\mu, \nu}(\alpha z)\right. \\
& \left.+\left(\frac{q_{-n}}{z^{n}}+\cdots+q_{0}+\cdots+q_{t} z^{t}\right) A_{\mu, \nu}^{\prime}(\alpha z)\right) e^{\alpha_{1} z},
\end{aligned}
$$

где $t \in \mathbb{Z}^{+}, p_{j}, q_{j} \in \mathbb{A}, j=-n, \ldots, t,\left(p_{-n}, q_{-n}\right) \neq(0,0)$. Так как правая часть равенства (32) должна быть целой функцией, то $p_{-n}+q_{-n} \nu / \mu=0$. Из уравнения (1) получаем $A_{\mu, \nu}(\alpha z)=(\mu / \nu-\alpha z / \nu) A_{\mu, \nu}^{\prime}(\alpha z)+(\alpha z / \nu) A_{\mu, \nu}^{\prime \prime}(\alpha z)$. Подставив это выражение в равенство (32) и учитьвая, что $A_{\mu, \nu}^{\prime}(\alpha z)=(\nu / \mu) A_{\mu+1, \nu+1}(\alpha z)$, получим

$$
\begin{aligned}
f(z)= & \left(\left(\frac{p_{-n+1}^{*}}{z^{n-1}}+\cdots+p_{0}^{*}+\cdots+p_{t+1}^{*} z^{t+1}\right) A_{\mu+1, \nu+1}(\alpha z)\right. \\
& \left.+\left(\frac{q_{-n+1}^{*}}{z^{n-1}}+\cdots+q_{0}^{*}+\cdots+q_{t+1}^{*} z^{t+1}\right) A_{\mu+1, \nu+1}^{\prime}(\alpha z)\right) e^{\alpha_{1} z},
\end{aligned}
$$

где $p_{j}^{*}, q_{j}^{*} \in \mathbb{A}, j=-n+1, \ldots, t+1$. Повторив эту процедуру не более чем $n$ раз, приходим к равенству

$$
f(z)=\left(P_{6} A_{\mu+m, \nu+m}(\alpha z)+P_{7} A_{\mu+m, \nu+m}^{\prime}(\alpha z)\right) e^{\alpha_{1} z},
$$

где $P_{6}, P_{7} \in \mathbb{A}[z], \alpha, \alpha_{1} \in \mathbb{A}, \mu, \nu \in \mathbb{Q}, m \in \mathbb{N}, m \leqslant n$. Следовательно, $f(z)$ имеет вид (2). Теорема 1 доказана.

\section{СПИСОК ЦИТИРОВАННОЙ ЛИТЕРАТУРЫ}

[1] Siegel C. L. Über einige Anwendungen Diophantischer Approximationen // Abh. Preuss. Acad. Wiss., Phys. -Math. Kl. 1929-1930. № 1. P. 1-70.

[2] Шидловский А. Б. Трансцендентные числа. М.: Наука, 1987.

[3] Горелов В. А. Об алгебраической независимости значений $E$-функций в особых точках и гипотезе Зигеля // Матем. заметки. 2000. Т. 67. № 2. С. 174-190.

[4] Кратцер А., Франц В. Трансцендентные функции. М.: ИЛ, 1963.

[5] Шидловский А. Б. О трансцендентности значений $E$-функций, являющихся решениями линейных дифференциальных уравнений второго порядка // Матем. сб. 1993. Т. 184. № 5. C. $75-84$.

[6] Камке Э. Справочник по обькновенным дифференциальным уравнениям. М.: Наука, 1971.

[7] Айнс Э. Л. Обыкновенные дифференциальные уравнения. Харьков, 1939.

[8] Стрелиц Ш. И. О росте решений линейных дифференциальных уравнений второго порядка // Матем. сб. 1958. Т. 46. № 4. С. 433-450.

[9] Виттих Г. Новейшие исследования по однозначным аналитическим функциям. М.: $\Phi \mathrm{M}, 1960$.

[10] Andre Y. Séries Gevrey de type arithmétique // Ann. of Math. 2000. V. 151. P. 705-756.

[11] Галочкин А. И. О критерии принадлежности гипергеометрических функций Зигеля классу E-функций // Матем. заметки. 1981. Т. 29. №1. С. 3-14. 